been studied in steady-study torsional shearing between two parallel circular disks and between a cone and a disk. Work continued on the development of new methods for obtaining accurate solutions of the molecular Schrödinger equation, and in motal physics tensile deformation investigations were carried out of the close-packed hexagonal copper-germanium phase, while the deformation stacking fault probability was measured in dilute intranoble metal alloys containing up to 5 atomic per cent solute. A list of publications is included.

\title{
WHEAT GENETICS
}

$\mathrm{T}$ HE second International Wheat Genetics Symposium was held during August 19-24 at the University of Lund, Sweden. The meetings produced a broad survey of many recent advances in knowledge of the genetical architecture of the hexaploid wheat of commerce, Triticum aestivum, and of some of its relatives in the genera Triticum, Aegilops, Agropyron and Secale. Naturally, considerable attention was directed to ways in which the new information could be exploited in the improvement of the crop, but since wheat offers many unique opportunities for genetical work there was no restriction to topics of immediate practical importance.

For example, R. C. F. Macer (Great Britain), R. G. Anderson (Canada), D. R. Knott (Canada) and A. T. Pugsley (Australia) considered the genetic control of re. sistance to stripe rust, leaf rust, stem rust and powdery mildew respectively. In the main, similar patterns of major gene effects are responsible for differences in resistance to all four fungal pathogens, and, as was pointed out by W. Q. Loegering (United States), alleles for resist. ance or susceptibility in the host could be matched by alleles for avirulence or virulence in the fungus, provided the latter had a sexual stage. Basic information of this type has a direct practical value since the recognition of the mode of inheritance greatly facilitates the use of genetically determined resistances in breeding programmes. Moreover, in all these instances the genes for resistance are being isolated, in otherwise equivalent genetic backgrounds giving full susceptibility, so that differential hosts will be available that will permit accurate assessments of the virulence genotypes of pathogens isolated in the field.

The practical importance of incorporating, in wheat, the genetically determined disease resistance of other species was widely accepted during the Symposium. The isolation against the wheat background was reported of single chromosomes from Agropyron giving resistance to stripe, stem and leaf rust by A. Wienhues (Germany), Y. Cauderon (France) and D. R. Knott (Canada), and of one giving resistance to the wheat streak mosaic virus by E. E. Sebesta (United States). R. C. F. Macer (Great Britain) and C. J. Driscoll (Australia) reported that single chromosomes of Secale, isolated against a wheat background, would give resistance to stripe rust and leaf rust. In several instances, use was being made of translocations induced by irradiation to transfer to a wheat chromosome the critical segment of the alien chromosome giving disease resistance.

A remarkable piece of engineering of this type was described by D. R. Knott (Canada). It involved the transfer of $S r 6$, a gene giving stem rust resistance carried on chromosome $2 D$ of the $D$ genome of $T$. aestivum, to $T$. durum, a tetraploid in which chromosome $2 D$ has no homologue. Chromosome $2 D$ was isolated, by backcrossing, against the $T$. durum background and a satisfactory translocation of $S r 6$ to a chromosome of $T$. durum was induced and made homozygous. Not only is a new form of resistance now available at the tetraploid. level, but also the Sr6-carrying tetraploid can be crossed to $T$. aestivum and the gene, in its new position, back-crossed into the hexaploid, and so obtained in four doses. Whether duplication of the gene will show a dose effect that improves disease resistance remains to be seen. A possible alternative to the introduction of alion variation by translocation might be to substitute a pair of alien chromosomes for a pair of wheat chromosomes. This can be achieved by sequences of hybridizations between various aneuploid lines. A. Wienhues (Germany) described the substitution of Agropyron pairs for wheat pairs, and B. C. Jenkins (Canada) the substitution of Secale pairs for wheat pairs. There was some discussion as to whether an alien pair could only be expected to replace a homoeologous wheat pair with which it had a common evolutionary origin, but insufficient evidence is as yet available to clarify the issue.

H. Kihara (Japan) described the production of male sterile forms of wheat by the substitution, by backcrossing, of the wheat nucleus into the cytoplasm of various other species, while J. W. Schmidt and V. A. Johnson (United States) outlined the discovery and use of genes that restore male fertility. This work clearly indicates that the commercial exploitation of hybrid wheat may soon be possible. It is not yet known whether there will be sufficient hybrid vigour to produce an increase in yield adequate to cover the increased cost of seed production - an increase in yield of about 20 per cent is estimated to be necessary. Moreover, many hold that genotypes giving the greater yields should be fixable by selection in the derivatives of hybrids showing heterosis, although this ignores the probable difficulties of breaking some repulsion linkages in a few generations of recombination.

The accumulation of conventional linkage data has been very slow in $T$. aestivum, but $\mathbf{E}$. R. Sears (United States) demonstrated how this could be remedied by using telocentric chromosomes to estimate linkage distances between single major genes and the centromere. In this way some linkage information has already been obtained about chromosomes $3 B$ and $6 B$. Although aneuploidy has been of the utmost value in the genetic analysis of $T$. aestivum, as was demonstrated by R. I. Larson (Canada) and J. Kuspira (Canada), it has been less useful with the tetraploid wheats-presumably because they have fewer duplicate loci and are consequently intolerant of aneuploidy. However, M. Noronha-Wagner and 'T. MelloSampayo (Portugal), working with T. durum (a tetraploid), have been able to hold the nullisomic or monosomic condition for some chromosomes provided that there was compensation for the deficiency by extra dosage of the homoeologue in the other genome. Of interest was the fact that there is some tendency for homoeologous chromosomes to pair at meiosis when in the monosomic-trisomic condition, despite the normally diploid-like meiotic behaviour of the tetraploid wheats.

R. Riley (Great Britain) provided confirmation of the hypothesis that homoeologous meiotic pairing and recombination occur when the restricted pairing specificity, imposed by the activity of chromosome $5 B$, is relaxed. Moreover, the relaxation of specificity permitted the incorporation in wheat chromosomes, by recombination, of segments from the chromosomes of other species in a manner that was otherwise impossible. In a parallel investigation of the $\operatorname{sam} \theta$ system, M. Okamoto (Japan) had selected induced mutants that altered the activity of chromosome $5 B$ in the regulation of meiosis. He indicated the likelihood that such mutants could be stabilized in stocks that would have considerable potential value in practical breeding work.

C. F. Konzak (United States) and R. E. Scossiroli (Italy) considered the value of induced mutations in wheat 
breeding and both emphasized the particular value of changes that affect quantitative characters. The genetic analysis of the protein quality of the grain was discussed in relation both to the physical causes of the different properties of flours and of their assessment in small-scale tests. Finally, no ultimate verdict was possible by $J$. Mac Key (Sweden) or M. S. Swaminatham (India) on the true nature of the genes on the pronounced effects of which are erected many taxonomic categories in Triticum.

Wheat is, with rice, the principal food crop of the world and to a considerable degree its present preeminence is owed to its extreme genetic versatility. The second International Wheat Genetics Symposium marked a further advance in our comprehension of this versatility and confirmed that the cytogenetic structure of $T$. aestivum continues to be the subject of intensive investigation in many parts of the world. Indeed there were several workers from the U.S.S.R. present, all of whom treated the genetics of wheat from the Lysenkoist point of view. That some of the fundamental cytogenetic investigations of the past have already been turned to practical account is gratifying, and promises well for the future of the more thorough understanding that we are now attaining.

RALPH RILEY

\section{TEACHING OF BIOLOGY}

\section{By DR. GARTH UNDERWOOD \\ University of the West Indies, Trinidad}

$\mathrm{T}$ HERE has been considerable recent discussion of the effect on the teaching of biology of the growing field in which the traditional distinction between botany and zoology is disappearing. Catcheside ${ }^{1}$ and Darlington ${ }^{2}$ put forward proposals for teaching the biological sciences which bring together microbes, plants, animals and man. Their proposals, however, still retain the divisions of biology into such aspects or "disciplines" as genetics, ecology, taxonomy and physiology.

These several biological disciplines grew up separately at a time when there was no general body of biological theory to relate them to one another. They started as inductive sciences. Investigators at this stage were trying to ask the right questions and to devise techniques to obtain the answers. Discussion of techniques figured largely in the discussion of the results and theories were fragmentary and controversial. The attention of each investigator was understandably turned in on his own field; at this stage other fields had little to offer him. This was the period during which there developed a deep gulf between field and laboratory. This period also produced some biologists who now look rather strange: the biochemist to whom a rat was the animal reared in the room at the end of the corridor, the taxonomer in a dusty museum whose study was, as Philip Gosse pointed out a hundred years ago, not so much biology as necrology, the geneticist who had no idea where to start looking for a Drosophila in the field, the physiologist who did not know the systematic relationships of (or sometimes even how to identify) the organisms which he studied. Parenthetically it may be remarked that this is perhaps why the origin of new adaptive features remains an obscure problem ${ }^{3}$. An account of the origin of a new feature would require a knowledge of its structure and function, of its genetical and developmental determination and of the habits and ecology of the organism; in other words, a synthesis of the disciplines which have usually been studied separately.

I would like to offer some suggestions aimed at achieving a greater merging of these several aspects of biology as well as a merging of plant and animal studies. We are still far from being able to proceed in a series of deductive steps from simple molecules to twentieth-century human society; however, we can now suggest the lines along which large areas of biology may be linked together. Organisms have been most studied as individuals. Bacteria, protista and higher plants and animals represent three grades of individual organization. Genetics has an important contribution to make to the discussion of cellular structure and function and, in the case of the higher organisms, to the organization of the development of the individual. Consideration of the higher organisms leads to the structural and physiological differentiation of tissues and organs. Some topics such as animal behaviour and locomotion remain frankly zoological. Such relationships as that between insect and flower, host and parasite, mimic and model or between the bees in a hive can be looked on as special cases of relationship between individuals.

Studied as individuals organisms can, however, be understood only in part. This leads us to the study of populations. A consideration of populations involves such topics as population dynamics, territory, living space, overt and concealed polymorphism, natural selection, geographic variation, reproductive isolation and the nature of species. The rules for dealing with lower taxonomic categories follow quite naturally on discussion of species. A number of special relationships already examined at the individual level may now be studied at the population level; insect and flower, host and parasite, mimic and model are viewed from a different angle.

From the relatively simple relationships between populations just mentioned we can proceed logically to the complexity of major communities. Here a number of ecological ideas such as niche, trophic-level and pyramid of numbers can be introduced. The grades of individual organization and the metabolism of plants and animals, already examined, now take on a new significance in the new context. From the examination of community structure we may proceed to a review of the adaptive diversity of plants and animals, a review of the niches which are actually filled.

Living organisms will now have been investigated as individuals, in populations and as members of communities. It remains for the student to recognize that recent living organisms have a long evolutionary history behind them: that the present cannot be fully understood without reference to the past. This leads us to consider temporal changes in the physical environment, the fossil record and the evidence on which phylogenetic schemes are constructed; here morphology has a major place and biogeography and the comparative approach to physiology, biochemistry and cytology can be considered. To present the findings of phylogenetic studies, rules for the treatment of higher taxa are necessary. The actual results of phylogenetic studies can now be reviewed in outline in the form of a historical survey of plants and animals and a classification of recent organisms.

Man will have furnished good illustrative material for some of the earlier parts of the course such as the nature of gene action, natural selection and population dynamics. An important part of the general educational value of a biology course, however, lies in the light which it throws on man himself. Such a course could well conclude by taking a look at man in the light of biological knowledge: the origin of man, genetics and physical anthropology, the domestication of plants and animals, population studies, food supplies, disease, radiation hazards and, finally, man in space.

The conventional 'packaging' of biological teaching made it easier for the student to leave out important 